\title{
Physiological Modeling and Control at Obuda University
}

\author{
L. Kovács*, T. Ferenci*, J. Sápi*, Gy. Eigner*, J. Klespitz, P. Szalay*, M. Kozlovszky**, I. Rudas* \\ ${ }^{*}$ Research and Innovation Center of Obuda University, Physiological Controls Group, Budapest, Hungary \\ \{kovacs.levente, ferenci.tamas\}@nik.uni-obuda.hu, \{sapi.johanna, eigner.gyorgy, klespitz.jozsef\}@phd.uni-obuda.hu, \\ szalay.peter.1986@gmail.com \\ ** Research and Innovation Center of Obuda University, BioTech Knowledge Center, Budapest, Hungary \\ $\{$ kozlovszky.miklos\}@nik.uni-obuda.hu \\ ${ }^{* * *}$ Research and Innovation Center of Obuda University, Antal Bejczy Center for Intelligent Robotics, Hungary \\ \{rudas\}@uni-obuda.hu
}

\begin{abstract}
The paper gives a summary of 2014 year results in the topic of physiological modeling and control achieved by the Physiological Controls Group of the Obuda University. The presentation is integrated in the tradition established years ago at IEEE INES conferences and 2014's IEEE SACI conference summarizing the latest results obtained by the group. Four topics are presented, highlighting collaboration with our partner research institutes as well: modern robust control of diabetes, antiangiogenic model-based tumor control, biostatistical modeling methods of diabetes, control of peristaltic pumps in hemodialyisis problem.
\end{abstract}

\section{INTRODUCTION}

In the biomedical research domain, experts typically face two major problems:

1) understanding the physiological behavior of the system and transform it into a (mathematical) model;

2) finding sufficiently accurate methods and sensory input modes to detect the required signals.

The aim of physiological modeling and control - a subdiscipline of biomedical engineering - is to study, model and understand biological processes, then to apply identification and control strategies on it as a system [1]. This approach enables the automated treatment of various diseases (ex. diabetes or cancer) and injuries of the human body (e.g. bio-prosthetics) by designing closed-loop control systems; hence, enabling personalized healthcare by giving the possibility to support medical devices for specific diseases or ease everyday life healthcare monitoring (i.e. by smartphone applications). Using control engineering methodologies (model identification and controller design) these protocols could become model-based.

The Physiological Controls Group of the Óbuda University performs researches in collaboration with together in control applications with the Department of Control Engineering and Information Technology of the Budapest University of Technology and Economics and the Semmelweis University from Budapest in the above mentioned topic focusing on four topics: modeling and control of diabetes, cancer, peristaltic pumps and biostatistical modeling. The current article summarizes the newest results obtained following the tradition established at previous IEEE INES and SACI conferences [2-5].

\section{DiABEtes Modeling, Estimation AND CONTROL}

Diabetes mellitus is a serious metabolic disease that should be artificially regulated. Due to the continuously increasing number of diabetic patients, the scientific community has aimed to improve the treatment of the disease by investigating the applicability of an external controller, so called artificial pancreas [6] (AP) that focus on Type 1 Diabetes Models (T1DM) having a general clinical symptom, the lack of insulin production of the patients.

Since T1DM models have usually nonlinear dynamics, the controllers used for blood glucose regulation are either nonlinear as well, or linear while consider the nonlinearity of the model by giving robust guarantees. An example for the latter is modern robust (e.g. $\mathrm{H}_{\infty}$ ) control. Robustness is necessary, as classical linear control methods (e.g. PID control [7]) faces difficulties ensuring the safety of the patient, and hence has limited practical use. There are several examples for the application of $\mathrm{H}_{\infty}$ control in blood glucose regulation [8]; however, as a linear control methodology.

\section{A. LMI-based robust LPV control}

We have focused on Linear Parameter Varying (LPV) based robust controllers making direct nonlinear control possible. Based on the developed robust control framework [9] we have investigates the capabilities of a robust LPV controller in order to check if it could be extended by additional constraints to answer certain practical issues, such as avoiding hypoglycemia and high carbohydrate intake.

Based on the model presented in [10] we have performed an LPV model synthesis on how to optimize scheduling parameter selection and address switching control challenges [10]. This investigation focused on weighting function determination and Linear Matrix Inequalities- (LMI-) based controller design (defining the problem as a convex constraint on the sought variables).

Results were tested on the 6 virtual patients of the Cambridge in-silico simulator [6], testing three different protocols: 2 days simulation with $150 \mathrm{~g}$ carbohydrate (CHO), 2 days simulation of the protocol based on [11], 2 days simulation with random meal timing unusually high (180g-310g) CHO intake [10]. We have concluded that the blood glucose can be maintained in the normoglycaemic region (Fig. 1). 


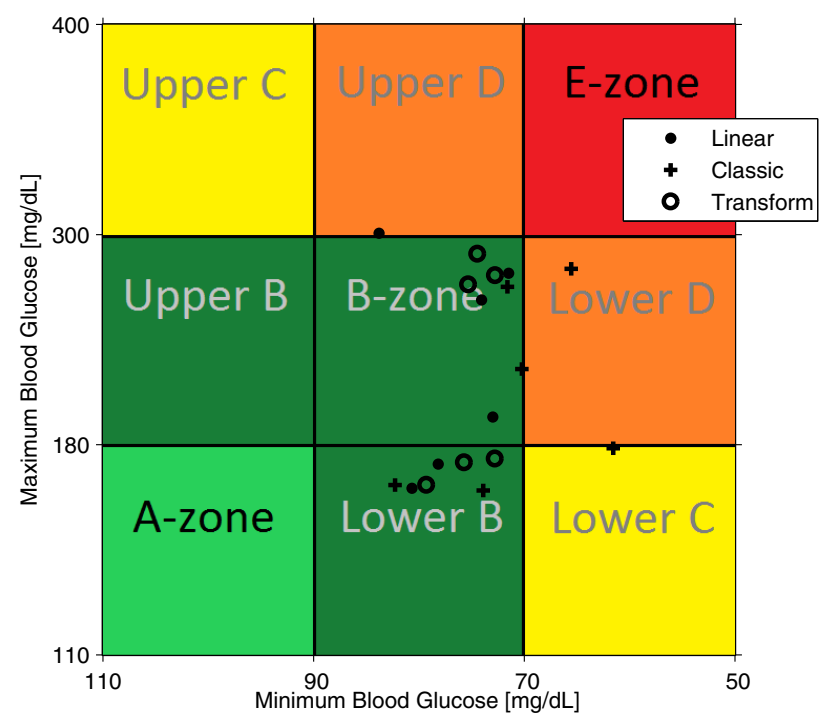

Figure 1. Simulation results of all LPV type controllers for the second meal intake scenario using the control variability grid analysis (CVGA) metric of [11].

\section{B. Estimation methods for sensor drift compensation}

LPV-based control has proven to be an adequate choice, but the time-varying parameters are needed to be available for measurement.

Model-based filters can satisfy the demands of estimating the non-observable states. In case of significant measurement noise and large unknown disturbances (like meal intake, physical activity) Kalman-filtering is usually preferred [12]. Extended Kalman Filter (EKF) is used for direct nonlinear model estimation, but it may fail if the system contains high nonlinearities or initial estimation errors. Sigma-point filters have been appeared in order to overcome these disadvantages [13]. We have implemented different sigma-point filter approaches (Cubature Kalman Filter (CKF), Unscented Kalman Filter (UKF), SparseGrid Quadrature Filter (SQKF)) and evaluated them using 500 simulations lasting 48 hours each, with randomized inputs, measurement noise and initial estimation [14]. The virtual patients had been taken from the same Cambridge in-silico simulator [6].

It was demonstrated (Fig. 2) that each investigated sigma-point filter proved to be better than the EKF. It was also proved that combining with $\mathrm{H}_{\infty}$ filtering method
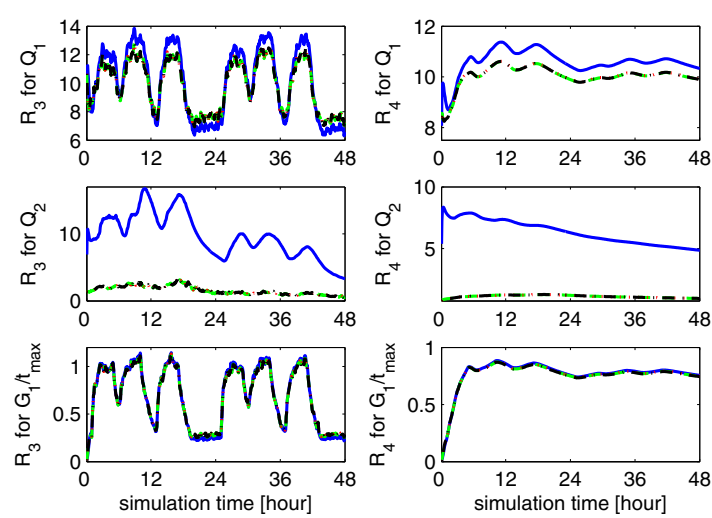

Figure 2. Estimation results of standard filters: EKF - solid line, CKF dashed line, UKF - dotted line, SQKF - dash-dotted line.
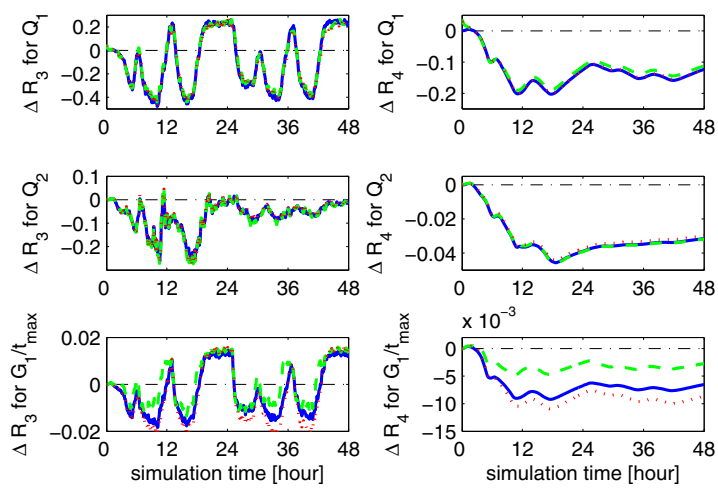

Figure 3. $\mathrm{H}_{\infty}$ filtering compared to regular CKF. CKF - solid line, $\mathrm{UKF}$ - dashed line, SQKF - dotted line, original CKF - dash-dotted line.

the results can even outperform the sigma-point filter results (Fig. 3) [14] that gives the possibility of extending the robust control framework with robust estimation considerations.

\section{BiostatisticAl Methods For GlyCAEMIC VARIABILITY INVESTIGATION}

Although T1DM can be treated by exogenous insulin intake blood glucose level of the diabetic patients exhibits higher-than-normal fluctuations, due to the lack of adequate control. It is already recognized that not only too low or too high glucose levels give rise to complications, but also too high glycemic variability itself [15].

The availability of continuous glucose monitoring (CGM) devices made it possible to track glycemic variability in a practically useful way. CGM systems usually have 5 minutes sampling frequency (as opposed to self-monitoring) which is sufficient to reconstruct the evolution of glucose level over time [16].

Even with such measurements available, it is still a question of how to define variability. Simple indicators such as the standard deviation are typically unable to grab to clinically relevant aspects of variability, thus, a myriad of more complex indicators were introduced [17]. HbA1c (glycolated hemoglobin) is also used in assessing the glycemic state of diabetic patients; however, it rather describes the average of the blood glucose to which the patient is exposed, and not its variability.

To investigate the connection between HbAlc (and also the basic characteristics of the patient: age and duration of diabetes) and different metrics quantifying a CGM recording, we carried out an empirical investigation taking into account 57 children with T1DM treated at the Department of Paediatrics of Semmelweis University (Budapest, Hungary). The majority of them were classified as being poorly controlled as per the guidelines of the American Diabetes Association and the International Society for Pediatric and Adolescent Diabetes. All patients underwent real-time (i.e. nonblinded) CGM recording. The abovementioned patient characteristics and $\mathrm{HbA} 1 \mathrm{c}$ levels were extracted from the electronic records of the hospital information system [18]. 
CGM recordings were electronically processed, and the following metrics were calculated:

- Relative time spent in hypo- and hyperglycemia;

- Mean and median glucose level;

- Standard deviation and interquartile range of glucose level;

- Mean Amplitude of Glycemic Excursions (MAGE).

These metrics and the patient characteristics - as all were continuous variables - were investigated with scatterplots and the calculation of correlation coefficients among each pair. To account for the possible non-linear (or even non-monotone) connections, distance correlation was also calculated.

Age and duration of T1DM showed no clear relationship with any descriptor of the glucose levels. HbA1c showed clear - and in many cases also statistically significant - positive correlation with several indicators of glycaemia. It is of particular interest that in several cases, the association was not monotone, with a temporary break at about $10 \% \mathrm{HbA} 1 \mathrm{c}$ (Fig. 4).

The interpretation of this phenomenon is not entirely clear; one possible explanation is that due to the poor adherence - which was characteristic for most of the children in the sample - the subjects temporarily (during the CGM measurement) increased their attention to glycemic control, which was made possible by the nonblinded nature of the CGM. This was possible only up to a certain point in glycemic state, after which, such compensation was no longer that effective. The temporarily altered behavior when subjects are aware of being monitored is already recognized in many fields of medicine, and is called the Hawthorne-effect. The small sample size and the convenience sampling of the present study limits the robustness of this conclusion, and further studies are needed to confirm this finding, and, if confirmed, investigate its determinants.

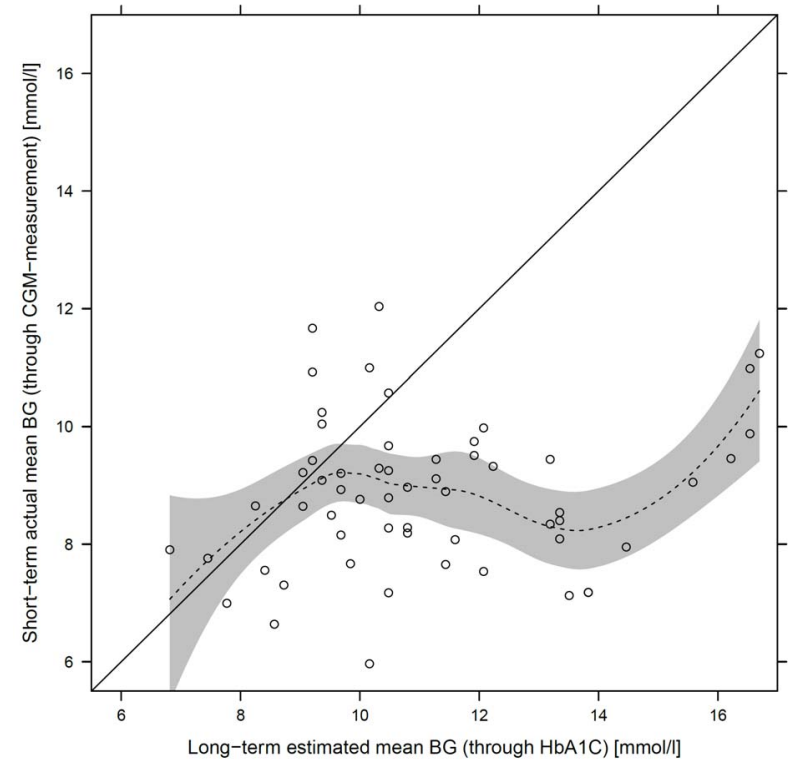

Figure 4. Converting HbA1c to glucose level ("long-term average glucose") and contrasting it with the average calculated from the CGM recording ("short-term average").

\section{AntiangIOGENiC TUMOR Growth MOdEL IDENTIFICATION AND CONTROL}

Our previous researches focused on development of control algorithms for antiangiogenic tumor therapy [5], [19] for the tumor growth model of [20]. However, based on our investigated inhibitors it turned out to identify a new tumor growth model.

The experiment has been done in collaboration with the 1st Department of Pathology and Experimental Cancer Research, Semmelweis University, Budapest, Hungary. We have carried out animal experiments to create and validate a clinically relevant tumor growth model, focusing on the effect of angiogenesis. There were several phases; each phase had a specific aim [21].

\section{A. C38 colon adenocarcinoma growth identification with and without Avastin therapy}

Parametric identification was carried out by fitting a curve with two exponential functions. The curve was fitted to the average tumor volume of each mouse at the measurement points (days). Comparison of the results in the three different cases can be found in Fig. 5. The coefficients of the exponential functions were positive in every case, reflecting the unstability of the system (as it is required from a tumor growth model). From the fitted curves, transfer function of the models has been calculated. From the poles of the systems (third row of Fig. 5) we can conclude that each system is unstable. To verify the goodness of the created transfer functions, we plotted the impulse response of each transfer function (second row of Fig. 5), which shows quite similar result to the curve fitting (first row of Fig. 5).

\section{B. Finding the relationship between tumor volume, mass and vascularization}

Three attributes of the lethal sized tumor were measured: tumor volume, tumor mass and vascularization. Relationship between these tumor attributes has been investigated with linear regression analysis. To decide whether the relationship is significant or not between two variables, we used Pearson correlation coefficient (R), coefficient of determination (R2) and Analysis of Variance (ANOVA) test (level of significance was chosen to $p=0.05)$. We have found that the relationship between tumor volume and mass is significant and positive in all cases, which means that the larger the volume, the higher the mass is. It has been demonstrated vascularization does not have significant relationship with volume or mass in neither cases.

\section{Finding the effective dosage for optimal therapy}

To compare the results of the three investigated experimental cases (Phase I, Phase III/2 control group, Phase III/2 case group) we used statistical analysis. Tumor volume values measured on the 21 st day in each phase have been compared. Normality was investigated with one-sample Kolmogorov-Smirnov test; it was found that each sample has normal distribution (ppI $=0.966$, $\mathrm{ppIII} 2 /$ control $=0.999, \mathrm{ppIII} 2 /$ case $=0.608)$. Homogeneity of variance was examined with Levene's test; the sample variances are equal $(p=0: 266)$. 

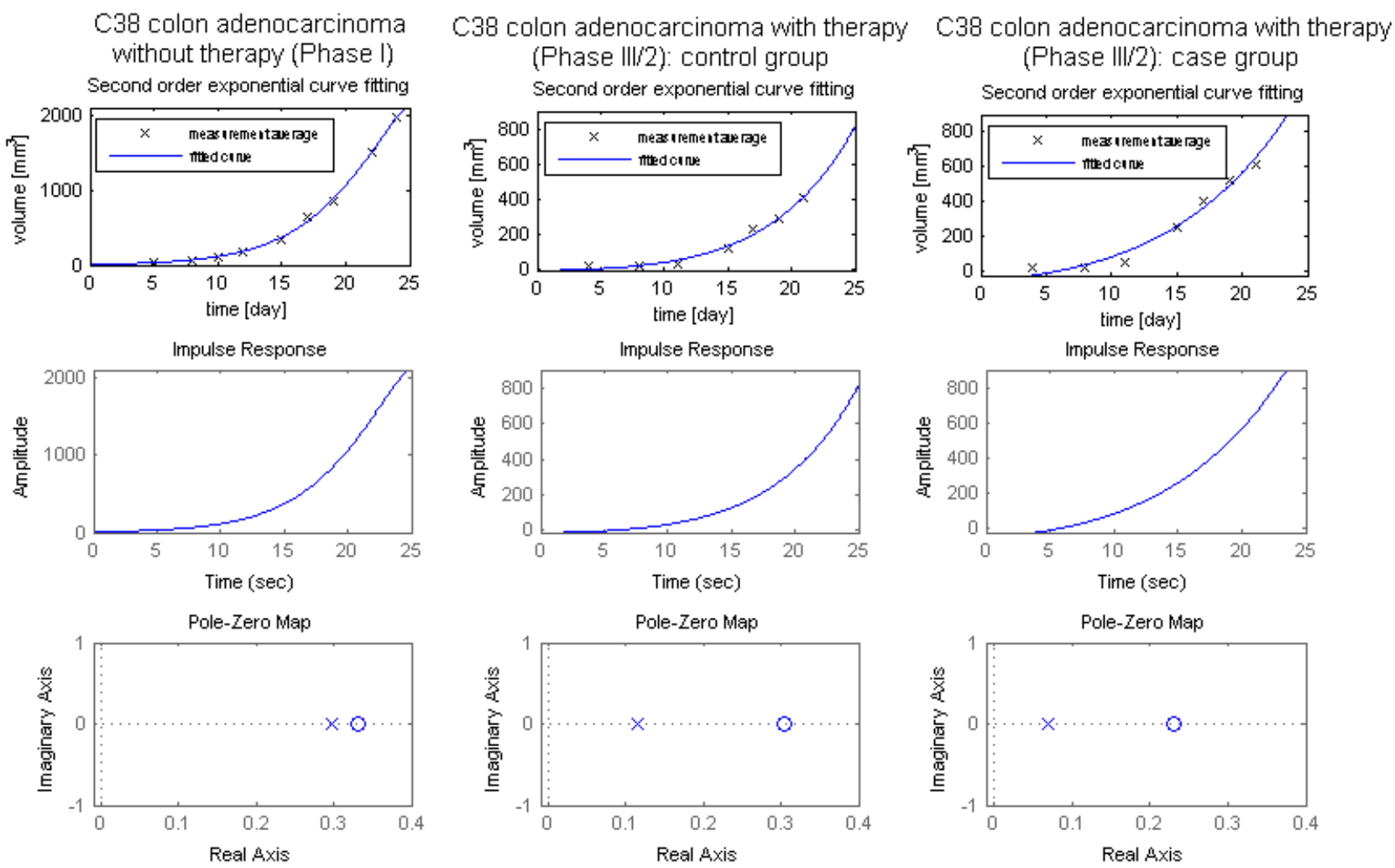

Figure 5. Comparison of $\mathrm{C} 38$ colon adenocarcinoma growth in three cases. The first row shows the second order exponential curve fitting for the average of measurement points; second row is for the impulse response of the identified systems; third row shows poles and zeros of the identified systems.

After confirming normality and homoscedasticity, parametric statistical analysis can be used. With Analysis of Variance (ANOVA) test more than two samples were comparable. ANOVA test was resulted in $p=0.038$ value meaning that we have to reject the null hypothesis according to which there are no differences between the means of the samples. To find those samples, which have significantly different means, Tukey's honest significant difference (HSD) test was used as post hoc test. The results are the following. Phase I and Phase III/2 control group are significantly different $(\mathrm{p}=0.034)$, which means that Avastin - administered according to the protocol - is an effective drug to reduce tumor volume. Phase III/2 control group and Phase III/2 case group are not significantly different $(p=0.416)$, however Phase I and Phase III/2 case group are not significantly different $(p=$ $0: 227)$ either. This means that the effectiveness of the "continuous" (daily) 1/180 dosage (1:11 $\mu \mathrm{g}$ relative to 200 $\mu \mathrm{g})$ is comparable with the effectiveness of one large dose.

\section{Peristaltic PUMP CONTROL OF Hemodialysis MACHINES}

A relevant problem of peristaltic pump is the inaccuracy of transfer. The error is resulted by the deviation of production, the fatigue of the material and the pressure. The inaccurate transfer may cause overdose in case of transfer of medical fluids. Furthermore, the inaccuracy may cause dehydration or water overdose in treated patient, who usually lack the function of the kidney (no urine production). In order to provide the necessary transfer volumes automatic control should be applied.
Furthermore, the controller has to be adaptive to react effectively on the changing environment, such as the changing pressure or the fatigue of material.

In previous studies [22], [23] a PID controller and a fuzzy-system were designed. These control methods were compared with a newly designed adaptive neuro-fuzzy inference system (ANFIS) [24]. The controllers were compared by settling time, overshoot and accuracy. From the view of patient the ideal controller is capable to correct the errors quickly (short settling time), with no unnecessary errors (no overshoot) introduced, and controls the system without the accumulation of errors (perfect accuracy). By comparing the controllers it was clear that the settling time of the examined controllers had only minor differences. The classical PID controller operated with overshoot, while the soft computing methods prevented the overshoot. The accuracy (Table I.) differs only slightly in the different controllers. All of the mentioned measurements were done with simulation, but they were also repeated with the real system. The operation of the hemodialysis machine proved the simulations are correct.

TABLE I. ACCURACY MEASUREMENT RESULTS

\begin{tabular}{|c|c|c|c|c|c|}
\hline $\begin{array}{c}\text { Accuracy } \\
{[\mathrm{g} * \mathrm{~s}]}\end{array}$ & $\begin{array}{c}100 \\
\mathrm{ml} / \mathrm{h}\end{array}$ & $\begin{array}{c}300 \\
\mathrm{ml} / \mathrm{h}\end{array}$ & $\begin{array}{c}500 \\
\mathrm{ml} / \mathrm{h}\end{array}$ & $\begin{array}{c}1000 \\
\mathrm{ml} / \mathrm{h}\end{array}$ & $\begin{array}{c}1500 \\
\mathrm{ml} / \mathrm{h}\end{array}$ \\
\hline PID & -1 & -1 & 1 & 0 & 1 \\
\hline Fuzzy System & -3 & 0 & -3 & -5 & -4 \\
\hline ANFIS & 0 & 2 & -1 & -2 & -2 \\
\hline
\end{tabular}




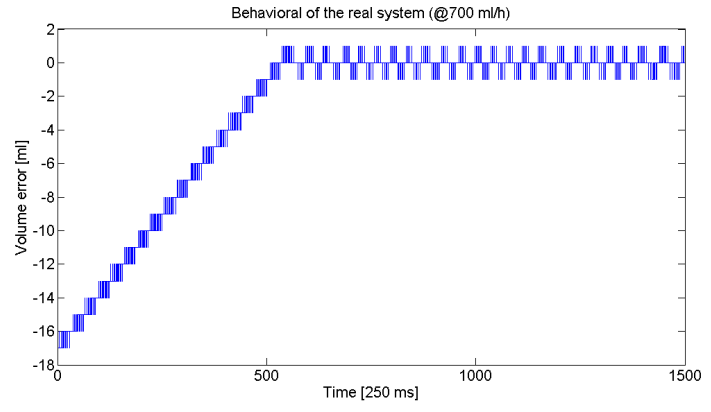

Figure 6. Behavior of the real system on the designed modified ANFIS controller.

The presented results confirm that soft-computing methods can be effectively used in safety-critical system and they have features compared to classical control methods capacitate them to apply in practice. Furthermore, the ANFIS system has the advantage that it is capable to adapt the changing environment.

\section{CONCLUSIONS}

The paper summarized the latest research results in the field of physiological modeling and control carried out by Physiological Controls Group of the Obuda University.

\section{ACKNOWLEDGMENT}

L. Kovács is Bolyai Fellow of the Hungarian Academy of Sciences. The authors say special thanks to the Hungarian Artificial Pancreas working group's insulin pump centers for the real data provided to validate the nonlinear model-based type 1 diabetes robust control algorithm as well as for Prof. Dr. László Barkai, president of the Hungarian Diabetes Association for his continuous support. For biostatistical evaluation data provision special thanks goes to Dr. Anna Körner from the Department of Paediatrics of Semmelweis University Budapest.

For antiangiogenic research task, the authors say special thanks to Dr. Zoltán Sápi, deputy head of the 1st Department of Pathology and Experimental Cancer Research Institute from Semmelweis University Budapest, and Dr. István Harmati and Dániel András Drexler from the Department of Control Engineering and Inormation Technology of the Budapest University of Technology and Economics.

The authors are grateful for the BBraun Medical Kft. for the support and for providing their real hemodialysis machine for measurements.

\section{REFERENCES}

[1] J. Bonzino, The Biomedical Engineering Handbook. CRC in cooperation with IEEE Press, 1995.

[2] L. Kovács, P. Szalay, T. Ferenci, D.A. Drexler, J. Sápi, I. Harmati, and Z. Benyó, "Modeling and Control Strategies of Diseases with High Public Health Impact", in Proc. IEEE INES 2011, Poprad, Slovakia, pp. 23-28, 2011.

[3] L. Kovács, P. Szalay, T. Ferenci, J. Sápi, P. Sas, D.A. Drexler, I. Harmati, B. Benyó and A. Kovács, "Model-based control algorithms for optimal therapy of high-impact public health diseases", in Proc. IEEE INES 2012, Lisabon, Portugal, pp. 531536, 2012.
[4] L. Kovács, J. Sápi, T. Ferenci, P. Szalay, D.A. Drexler, Gy. Eigner, P.I. Sas, B. Kiss, I.H. Harmati, M. Kozlovszky, and Z. Sápi, "Model-based optimal therapy for high-impact diseases", in Proc. IEEE INES 2013, San Jose, Costa Rica, pp. 209-214, 2013.

[5] L. Kovács, J. Sápi, Gy. Eigner, T. Ferenci, P. Szalay, J. Klespitz, B. Kurtán, M. Kozlovszky, D.A. Drexler, P. Pausits, I. Harmati, Z. Sápi and I. Rudas, "Model-based healthcare applications at Obuda University", in Proc. IEEE SACI 2014, Timisoara, Romania, pp. 183-187, 2014.

[6] W. E. Wilinska, L. J. Chassin, C. L. Acerini, J. M. Allen, D. B. Dunger and R. Hovorka, "Simulation environment to evaluate closed-loop insulin delivery systems in type 1 diabetes, J Diab Sci Techn, vol. 4(1), pp. 132-144, 2010.

[7] C. C. Palerm, "Physiologic insulin delivery with insulin feedback: A control systems perspective", Comp Meth Progr Biomed, vol. 102(2), pp. 130-137, 2011.

[8] P. Colmegna and R. S. Sanchez Pena, "Analysis of three T1DM simulation models for evaluating robust closed-loop controllers", Comp Meth Progr Biomed, vol. 113(1), pp. 371-382, 2014.

[9] L. Kovács, P. Szalay, Zs, Almássy, L. Barkai, "Applicability Results of a Nonlinear Model-Based Robust Blood Glucose Control Algorithm", J Diab Sci Techn, vol. 7(3), pp. 708-716, 2013.

[10] P. Szalay, Gy. Eigner, L. Kovács, “ Linear Matrix Inequalitybased Robust Controller design for Type-1 Diabetes Model", in Proc. 19th IFAC WC, Cape Town, South Africa, pp 9247-9252, 2014.

[11] L. Magni, D. Raimondo, C. Dalla Man, M. Breton, S. Patek, G. de Nicolao, C. Cobelli, and B. Kovatchev, "Evaluating the efficacy of closed-loop glucose regulation via control-variability grid analysis", J Diab Sci Techn, vol. 2(4), pp. 630-635, 2008.

[12] S..Haykin, Kalman Filtering and Neural Networks. John Wiley \& Sons, 2002.

[13] I. Arasaratnam and S. Haykin, "Cubature kalman filters," IEEE T Aut Contr, vol. 54(6), pp. 1254-1269, 2009.

[14] P. Szalay, A. Molnár, M. Müller, Gy. Eigner, I. Rudas, Z. Benyó and L. Kovács, "Comparison of Sigma-Point Filters for State Estimation of Diabetes Models", in Proc. IEEE SMC 2014, San Diego, USA, pp. 2506-2511, 2014.

[15] The Diabetes Control and Complications Trial Research Group, "The effect of intensive treatment of diabetes on the development and progression of long-term complications in insulin-dependent diabetes mellitus", New Engl J Med, vol. 329, pp.977-986, 1993.

[16] H. A. Wolpert, "Continuous glucose monitoring - coming of age", New Engl J Med, vol. 363, pp. 383-384, 2010.

[17] K.D. Kohnert, P. Heinke, L. Vogt and E. Salzsieder, "Utility of different glycemic control metrics for optimizing management of diabetes", World J Diabetes, vol. 6(1), pp. 17-29, 2015.

[18] T. Ferenci, A. Körner and L. Kovács, "The interrelationship of $\mathrm{HbA} 1 \mathrm{c}$ and real-time continuous glucose monitoring in children with type 1 diabetes", Diabetes Res Clin Pract, vol. 108(1). pp. 38-44, 2015.

[19] L. Kovács, A. Szeles, J. Sápi, D.A. Drexler, I. Rudas, I. Harmati and Z. Sápi. "Model-based Angiogenic Inhibition of Tumor Growth using Modern Robust Control Method", Comp Meth Prog Biomed, vol. 114, pp. 98-110, 2014.

[20] P. Hahnfeldt, D. Panigrahy, J. Folkman, and L. Hlatky, "Tumor development under angiogenic signaling: A dynamical theory of tumor growth, treatment response, and postvascular dormancy", Cancer research, vol. 59, pp. 4770-4775, 1999

[21] J. Sápi, D. A. Drexler, Z. Sápi and L. Kovács, "Identification of C38 colon adenocarcinoma growth under bevacizumab therapy and without therapy", in Proc. IEEE CINTI 2014, Budapest, Hungary, pp. 443-448, 2014.

[22] J. Klespitz and L. Kovács, "Identification and control of peristaltic pumps in hemodialysis machines", in Proc. IEEE CINTI 2014, Budapest, Hungary, pp. 83-87, 2013.

[23] J. Klespitz, M. Takács and L Kovács, "Application of fuzzy logic in hemodialysis equipment", in Proc. IEEE INES 2014, Tihany, Budapest, pp. 169-173, 2014.

[24] J. Klespitz, M. Takács, I. Rudas and L. Kovács, "Adaptive soft computing methods for control of hemodialysis machines", in Proc. iFUZZY 2014, Kaoshiung, Taiwan, pp. 109-112, 2014. 